\title{
Editorial: Gender Differences in Cardiovascular Diseases
}

\author{
Qiulian Zhou $^{1,2} \cdot$ Yihua Bei $^{2}$
}

Published online: 15 January 2020

(C) Springer Science+Business Media, LLC, part of Springer Nature 2020

There is increasing evidence that gender-related differences exist in cardiovascular diseases (CVDs). Males and females have certain differences in cardiac structure and function, sex hormones, and socio-psychological characters, thus leading to disparities in the susceptibility to and development of CVDs, clinical treatment and medical care, and outcomes of patient $[1,2]$. However, most of clinical strategies are based on studies with an under-representation of women. Studies focusing on gender differences in CVDs are highly needed, which may promote gender-specific diagnosis, treatment, and prognosis of CVDs.

This special issue entitled "Gender Differences in Cardiovascular Diseases" contains 8 selected papers dedicated to the influence of gender on cardiovascular physiology, pathophysiology, and diseases such as hypertension, cardiac hypertrophy, coronary artery diseases, arrhythmias, and heart failure. Gender-specific aspects of CVDs have been carefully reviewed and discussed in the context of prevalence, clinical characteristics, diagnosis, treatment, and prognosis.

In this special issue, Zhang et al. first gave an overview of the influence of gender on cardiovascular physiology as well as the pathophysiological changes during cardiac hypertrophy, fibrosis, arrhythmia, and coronary lesions. They also summarized gender-related differences in various cardiovascular risk factors, clinical symptoms, responses to treatment, and outcomes of patients [3]. Epidemiological studies have demonstrated that females normally have a later onset of CVDs than males, which is supposed to be related to the regulatory effect of estrogen on basic physiology in the cardiovascular system [4]. In addition to estrogen, testosterone is also an important

Yihua Bei

beiyh36@shu.edu.cn

1 Shanghai Applied Radiation Institute, School of Environmental and Chemical Engineering, Shanghai University, Shanghai 200444, China

2 Cardiac Regeneration and Ageing Lab, Institute of Cardiovascular Sciences, School of Life Science, Shanghai University, 333 Nan Chen Road, Shanghai 200444, China sex hormone. Lorigo et al. summarized the association of testosterone with multiple risk factors of CVDs, such as type 2 diabetes mellitus, obesity, hypertension, and dyslipidemia. The relationship between the reduced testosterone level and the pathophysiology of vascular dysfunction, coronary arterial diseases, heart failure, ischemic stroke, and atrial fibrillation has also been discussed [5]. These papers provide a very good overview to enhance our knowledge of gender differences in CVDs.

A series of papers were then dedicated to giving more focus on gender-specific differences in hypertension, cardiac hypertrophy, and arrhythmias. Song et al. overviewed gender differences in different types of hypertension, and also summarized the mechanisms such as angiotensin II and endothelin signaling, sex hormones, sympathetic nervous activity, and immunomodulation that contribute to gender differences in hypertension [6]. Wu et al. provided a comprehensive review of the roles of gender in both pathological and physiological cardiac hypertrophy and described sex-specific hormone factors and molecular networks in the regulation of cardiac hypertrophy [7]. Tian et al. systemically reviewed gender differences in cardiac arrhythmias, with a special emphasis on the most common type of arrhythmia atrial fibrillation (AF). In addition to epidemiology, risk factors, and clinical symptoms, the authors also described gender-related differences in electrical and structural remodeling of AF and different complications and outcomes of AF [8].

Multiple lines of evidence have proved gender differences in coronary arterial diseases, however, less attention has been paid for the preferentially higher prevalence of nonobstructive coronary arterial disease in females $[9,10]$. Groepenhoff et al. described gender-related difference in the structural and functional changes of coronary microvascular disease and also discussed the potential cellular and molecular mechanisms. They also provided updated information about functional assessment methods of the coronary microcirculation and indicated the importance of identifying genderspecific cut-off values for diagnosis and evaluation of coronary microvascular disease [11]. 
Finally, gender-specific prognostic markers of CVDs have been reported by 2 groups. Wu et al. analyzed a cohort of patients with acute heart failure (AHF) that were followed up for 18 months and identified different independent prognostic markers of all-cause death in males and females [12]. As an important etiology of heart failure, myocardial infarction is highly associated with recurrent cardiovascular events. Wang et al. reported that in patients with acute myocardial infarction (AMI), males and females differ in predictive markers of hospital readmission and/or death during a 6month follow-up [13]. A deep understanding of the mechanisms on how these predictive markers influence the prognosis of CVDs will further improve treatment and care of male and female patients.

Collectively, this special issue provides an overview and updated knowledge of gender differences in CVDs. More studies including clinical trials and animal experiments will be needed to further elucidate the influence and mechanism of gender-related differences in CVDs. Gender-specific aspects should be paid more attention to the evaluation and management of patients with CVDs.

Funding Information Our work was supported by the grants from the National Natural Science Foundation of China (81770401, 81970335 to Y.B.), Shanghai Rising-Star Program (19QA1403900 to Y.B.), and National Key Research and Development Program of China (2017YFC1700400, 2017YFC1700401).

\section{Compliance with Ethical Standards}

Conflict of Interest The authors declare that they have no conflict of interest.

Research Involving Human Participants and/or Animals This article does not contain any studies with human participants or animals performed by any of the authors.

Informed Consent This article does not contain any studies with human participants.

\section{References}

1. Mosca, L., Ferris, A., Fabunmi, R., Robertson, R. M., \& American Heart, A. (2004). Tracking women's awareness of heart disease: an
American Heart Association national study. Circulation, 109(5), 573-579.

2. Mehta, L. S., Beckie, T. M., DeVon, H. A., Grines, C. L., Krumholz, H. M., Johnson, M. N., et al. (2016). Acute myocardial infarction in women: a scientific statement from the American Heart Association. Circulation, 133(9), 916-947.

3. Zhang, Y., Liu, B., Zhao, R., Zhang, S., Yu, X. Y., \& Li, Y. (2019). The influence of sex on cardiac physiology and cardiovascular diseases. Journal of Cardiovascular Translational Research. https:// doi.org/10.1007/s12265-019-09898-x.

4. Murphy, E., \& Steenbergen, C. (2014). Estrogen regulation of protein expression and signaling pathways in the heart. Biology of Sex Differences, 5(1), 6.

5. Lorigo, M., Mariana, M., Oliveira, N., Lemos, M. C., \& Cairrao, E. (2019). Vascular pathways of testosterone: clinical implications. J Cardiovasc Transl Res. https://doi.org/10.1007/s12265-019-099395.

6. Song, J. J., Ma, Z., Wang, J., Chen, L. X., \& Zhong, J. C. (2019). Gender differences in hypertension. Journal of Cardiovascular Translational Research. https://doi.org/10.1007/s12265-01909888-z.

7. Wu, J., Dai, F., Li, C., \& Zou, Y. (2019). Gender differences in cardiac hypertrophy. Journal of Cardiovascular Translational Research. https://doi.org/10.1007/s12265-019-09907-z.

8. Tian, X. T., Xu, Y. J., \& Yang, Y. Q. (2019). Gender differences in arrhythmias: focused on atrial fibrillation. J Cardiovasc Transl Res. https://doi.org/10.1007/s12265-019-09918-w.

9. Hvelplund, A., Galatius, S., Madsen, M., Rasmussen, J. N., Rasmussen, S., Madsen, J. K., et al. (2010). Women with acute coronary syndrome are less invasively examined and subsequently less treated than men. European Heart Journal, 31(6), 684-690.

10. Yahagi, K., Davis, H. R., Arbustini, E., \& Virmani, R. (2015). Sex differences in coronary artery disease: pathological observations. Atherosclerosis, 239(1), 260-267.

11. Groepenhoff, F., Bots, S. H., Kessler, E. L., Sickinghe, A. A., Eikendal, A. L. M., Leiner, T., et al. (2019). Sex-specific aspects in the pathophysiology and imaging of coronary macro- and microvascular disease. Journal of Cardiovascular Translational Research. https://doi.org/10.1007/s12265-019-09906-0.

12. Wu, X., Chen, M., Wang, K., Gao, R., \& Li, X. (2019). Gender differences in prognostic markers of all-cause death in patients with acute heart failure: a prospective 18-month follow-up study. Journal of Cardiovascular Translational Research. https://doi.org/ 10.1007/s12265-019-09893-2.

13. Wang, P., Yao, J., Xie, Y., \& Luo, M. (2019). Gender-specific predictive markers of poor prognosis for patients with acute myocardial infarction during a 6-month follow-up. Journal of Cardiovascular Translational Research. https://doi.org/10.1007/ s12265-019-09946-6.

Publisher's Note Springer Nature remains neutral with regard to jurisdictional claims in published maps and institutional affiliations. 